\title{
A case of severe hypercalcaemia
}

\author{
V Arul Devah, X Lee, V Shetty, P Turner, K Imtiaz, S Howell, K Kaushal \\ Departments of Endocrinology and Surgery \\ Lancashire Teaching Hospitals Foundation Trust, Sharoe Green Lane, Preston, Lancashire
}

\section{Introduction}

Primary hyperparathyroidism affects about $0.3 \%$ of the general population. ${ }^{[1]}$

Approximately $80 \%$ of cases are related to the presence of a single parathyroid adenoma. ${ }^{[1]}$

Whilst parathyroidectomy remains the only curative approach, medical therapy with cinacalcet is extremely effective in controlling hypercalcaemia in the majority of patients. ${ }^{[2-4]}$

- Intravenous bisphosphonate is a well-established treatment for severe hypercalcaemia ${ }^{[5]}$

- We present a case of very severe and resistant hypercalcaemia secondary to primary hyperparathyroidism.

\section{Case History}

A 74 year old gentleman was admitted with a short history of confusion and reduced mobility.

He was noted to have a raised serum corrected calcium (cCa) of greater than $5 \mathrm{mmol} / \mathrm{L}$.

- An initial parathyroid hormone (PTH) level was $1.9 \mathrm{pmol} / \mathrm{L}$ (range 1.6-6.8) and he had acute kidney injury.

- He had features of sepsis and received antibiotics.

- He was rehydrated and given a dose of iv zoledronic acid. A repeat calcium 2 days after bisphosphanate therapy was $4.39 \mathrm{mmol} / \mathrm{L}$. He underwent dialysis and serum calcium improved transiently, the nadir value being $2.94 \mathrm{mmol} / \mathrm{L}$.

- A myeloma screen was negative. Computed tomography of the thorax, abdomen and pelvis demonstrated no evidence of malignancy, but showed features suggestive of acute pancreatitis.

- It emerged that he had presented to his local hospital 7 years ago with similar symptoms. Serum calcium had been elevated at $5.53 \mathrm{mmol} / \mathrm{L}$. He received acute treatment for hypercalcaemia, but refused further assessment.

- A repeat PTH level, checked after haemodialysis and 2 doses of zoledronic acid, was significantly elevated at $120 \mathrm{pmol} / \mathrm{L}$ (cCa $2.95 \mathrm{mmol} / \mathrm{L})$.

- A diagnosis of primary hyperparathyroidism was made at this stage and imaging organised.

- A second dose of bisphosphonate resulted in minimal lowering of serum cCa and he was commenced on cinacalcet and intravenous fluids. A third dose of bisphosphanate again resulted in only a transient improvement in hypercalcaemia.

- Figure 1 illustrates the changes in serum cCa levels in this patient and the therapies given.

- A sestamibi scan was felt to be possibly suggestive of a left inferior parathyroid adenoma (Figure 2).

- A parathyroid ultrasound demonstrated a $1.7 \mathrm{~cm}$ by $1.6 \mathrm{~cm}$ by $3.2 \mathrm{~cm}$ adenoma at the inferior pole of the left thyroid lobe (Figure 3).

- He was referred for a surgical opinion and underwent inpatient parathyroidectomy. He required further dialysis pre-operatively as his serum cCa was still around $3.7 \mathrm{mmol} / \mathrm{L}$ (PTH 205pmol/L).

- Post-operatively, serum calcium normalised and he then became hypocalcaemic, necessitating iv calcium gluconate.

- Parathyroid histology confirmed a $3.5 \mathrm{~cm}$ by $2.2 \mathrm{~cm}$ by $1.5 \mathrm{~cm}$ adenoma.

- He was hypocalcaemic at discharge and subsequently defaulted from outpatient follow-up.
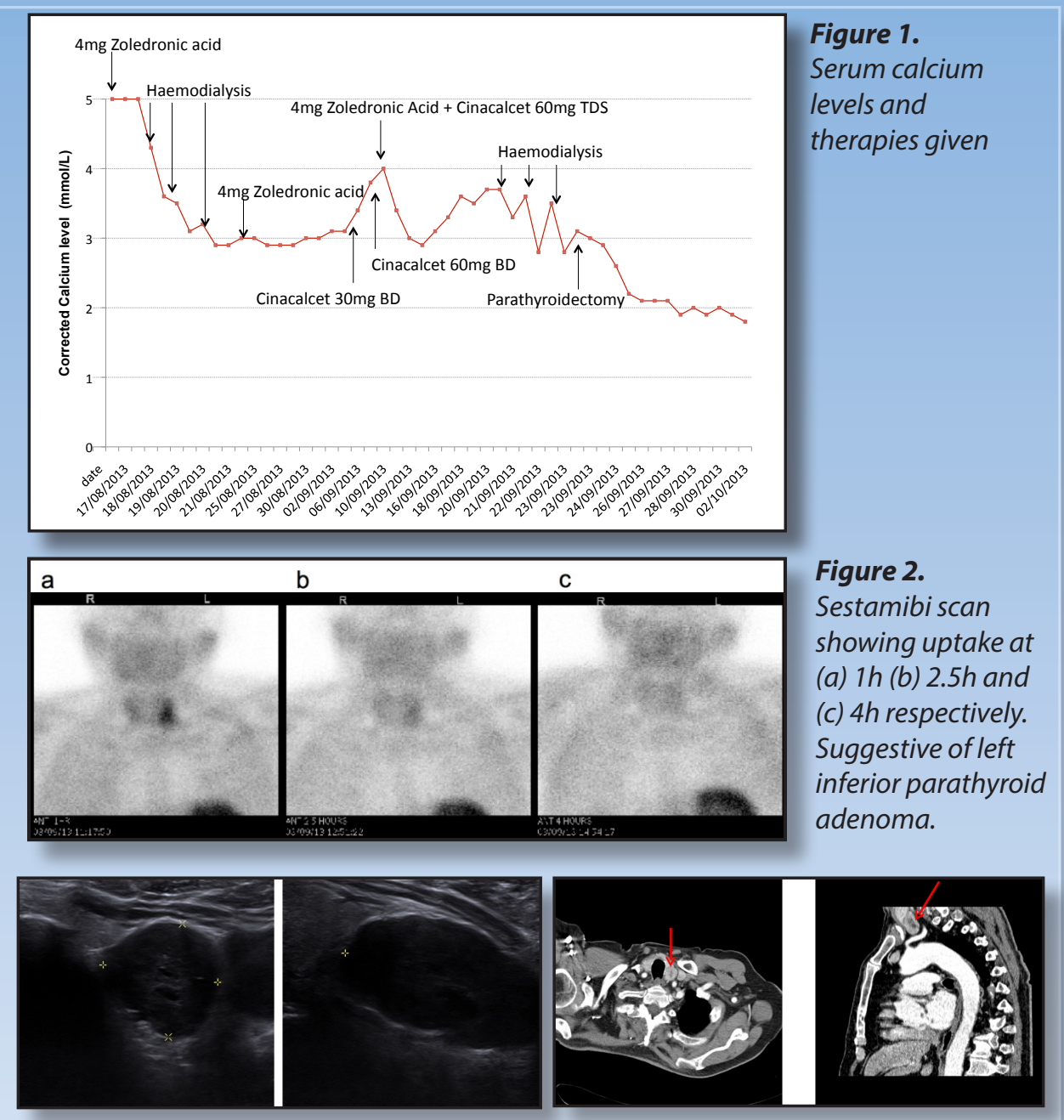

Figure 3.

Parathyroid ultrasound scan showing the left inferior parathyroid adenoma

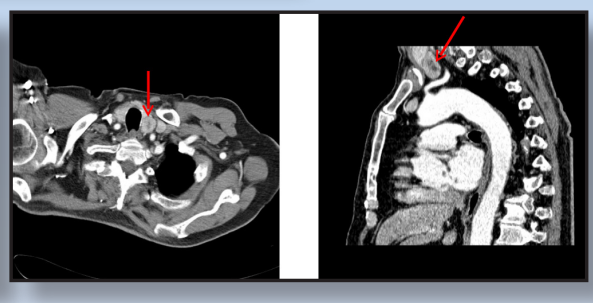

Figure 4.

CT scan with red arrows indicating parathyroid adenoma

\section{Discussion}

This case presentation highlights the clinical challenges we faced in managing this gentleman's hypercalcaemia.

Despite administration of cinacalcet and repeated doses of bisphosphonate, his serum calcium remained elevated without a sustained improvement.

His PTH was at the lower end of the reference range at presentation, subsequently becoming significantly elevated. This initial low PTH level led to possibly unnecessary investigation to rule out malignancy. However retrospective review of his $\mathrm{CT}$ thorax/abdomen/pelvis did in fact reveal the presence of the parathyroid adenoma (Figure 4).

The reason for the low admission PTH is not clear. Other than the possibility of an assay issue, one could speculate that his hypercalcaemia was so severe and prolonged that some degree of PTH suppression occurred.

In conclusion, primary hyperparathyroidism may occasionally be associated with very severe hypercalcaemia resistant to standard therapies. Once the diagnosis is established, urgent referral for inpatient parathyroidectomy may reduce the morbidity associated with this condition.

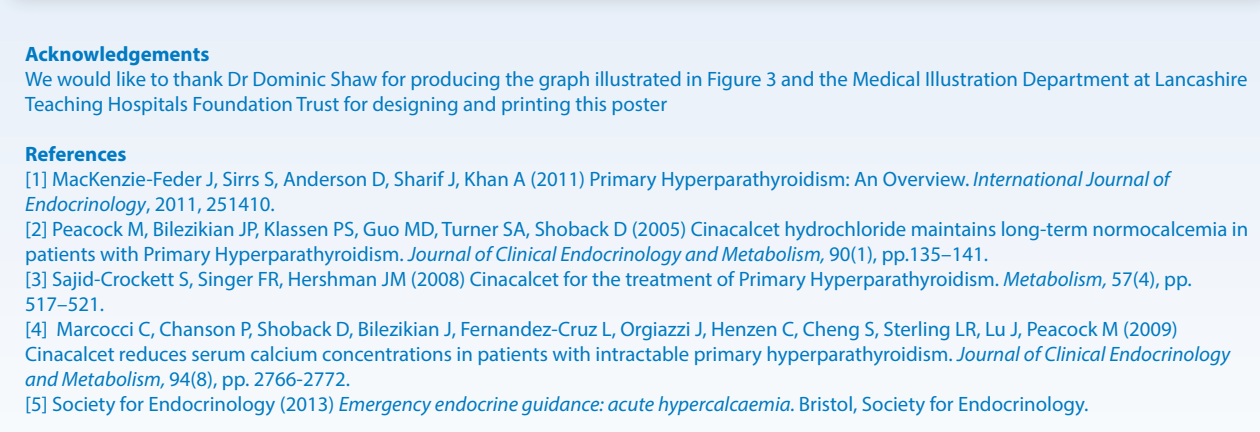
NHS Foundation Trust 